\title{
Industrial Internet of Things (IIoT) - An IoT Integrated Services for Industry 4.0 : A Review
}

\author{
Joyce Jacob $\mathbf{J}^{1}$ and Thamba Meshach $\mathbf{W}^{2}$
}

${ }^{I}$ Department of Electronics and Communication Engineering, Prince Dr. K. Vasudevan College of Engineering and Technology, Chennai, India

${ }^{2}$ Department of Computer Science and Engineering, Prathyusha Engineering College, Chennai, India

*Corresponding author: drjoycejacob@gmail.com / twmeshach@gmail.com

\begin{abstract}
Internet of Things (IoT), an inter-connecting technology comprising devices, objects, information, people and data, as a global internetwork for building intelligent systems. Such systems play a vital role in automating data sensing, machine and manufacturing process monitoring, product quality checking and lactation based smart shipping in real world industrial environment with integrity and interoperability. Industrial Internet of Things (IIoT) provides the integrated development environment for industries to build intelligent interconnected systems that uses the various IoT devices and bring the cyber and physical world together with higher level of availability and scalability. In this paper, we reviewed the transition of various IoT based systems to IIOT with the insights of concepts, devices and technologies of IoT and IIoT integrated applications which outlines the various trends and applications of Industry 4.0.
\end{abstract}

Keywords: Industrial IIoT, Industry 4.0, IoT Integrated service, Smart factory

In recent years a huge amount of work has been carried out in IoT for real-life application. It is defined as a technology that interconnects physical devices along with its inter-relation of data via internet without intervene of human or human to human interaction ${ }^{[1-2]}$. The idea was often known to be as "embedded internet"[3]. But the actual term "internet of things" was introduced by Kevin Ashton in 1999. In 2011, Gartner, the market research company included a new emerging phenomenon on their list: "The Internet of Things"[4]. The IoT supports to build systems for commercial sector, and IIoT for the industrial sector. IIoT technology made it possible to integrate all this data - sensor output, user input, service provider availability and knowledge to provide accurate and effective real-time responses.

Due to the usage of smart devices, mobiles phones, laptops have expanded so too the internet of things. 
IIoT devices are installed in industry / machinery environment to sense huge amount of data from a productive system which can be analyzed to understand, monitor and control the underlying events in an effective way. In IIoT, devices can be installed for a dedicated purpose (fixed) or re-configurable where the device setting can be changed w.r.t its sensing parameters and it is re-used for a similar but different purpose. In support of computing technologies, IIoT eases the mutual exchange of data or information between the users and the industrial equipment for service support and effective solutions to user problems ${ }^{[5]}$. Different kinds of smart applications are supported by IIoT such as smart city, smart houses, smart farming and smart factory and manufacturing ${ }^{[6]-[8]}$.

IIoT applications require relatively small throughput per node without the concern on the capacity. Instead, the need of connecting internet with a very large number of devices at low cost, with limited hardware capabilities and efficient energy resources, security, reliability, and cost more desired features ${ }^{[9]}$.

\section{IoT - IIoT}

IoT connects the physical things or devices through wired and wireless network whereas, IIoT deals with the complex physical machinery which are connected with industrial sensors and its relevant software. Industrial internet of things connects machines with human-interfacing unit for error-free system ${ }^{[10]}$. IoT focuses on the design, connect and communication standards into the Internet ecosystem through a friendly and flexible end- user application. In contrast, the IIoT focuses on integration and interconnection of remote plants and production machineries, thus offering a more efficient predictive production and services. For this reason, IIoT can be considered more an evolution rather than a revolution, in comparison with IoT. Table I gives a comparison of IoT and IIoT in the perspective of the technologies sector, model, data volume, communication and connectivity model.

Table 1: Comparison on Internet of Things (IoT) and Industrial IoT (IIOT)

\begin{tabular}{ll}
\hline IOT & IIOT \\
\hline IoT for Commercial Sector & IIoT for Industrial Sector \\
Human centred model & Machine centred Model \\
Volume of data is Medium & Volume of data is very High \\
Machine to Human communication. & Machine to Machine(M2M) communication. \\
$\begin{array}{l}\text { Supports Infrastructure Less Mobile Ad-hoc } \\
\text { connectivity }\end{array}$ & $\begin{array}{l}\text { Structured Infrastructure based centralised connectivity } \\
\text { is required. }\end{array}$ \\
Human and Things integrated Technology & $\begin{array}{l}\text { Machine-to-Machine Integrated Digital Manufacturing } \\
\text { and Operational Technology }\end{array}$ \\
\hline
\end{tabular}

\section{IIoT ARCHITECTURE}

The structure of a IIoT architecture highlights scalability, modularity, and interoperability with various devices and platforms using different technologies. Recently, the "Reference Architecture" document ${ }^{[1]}$ released by the Industrial Internet Consortium, which highlights on different viewpoints on business, usage, functional and implementation views and provides models. Technologies and the system components were focused in implementation viewpoint, and it describes the components structure and the interconnecting 
topology and the protocol stack of the architecture. IIoT mainly focuses on the transfer and control of mission critical information and responses, and relies on machine-to-machine communications.

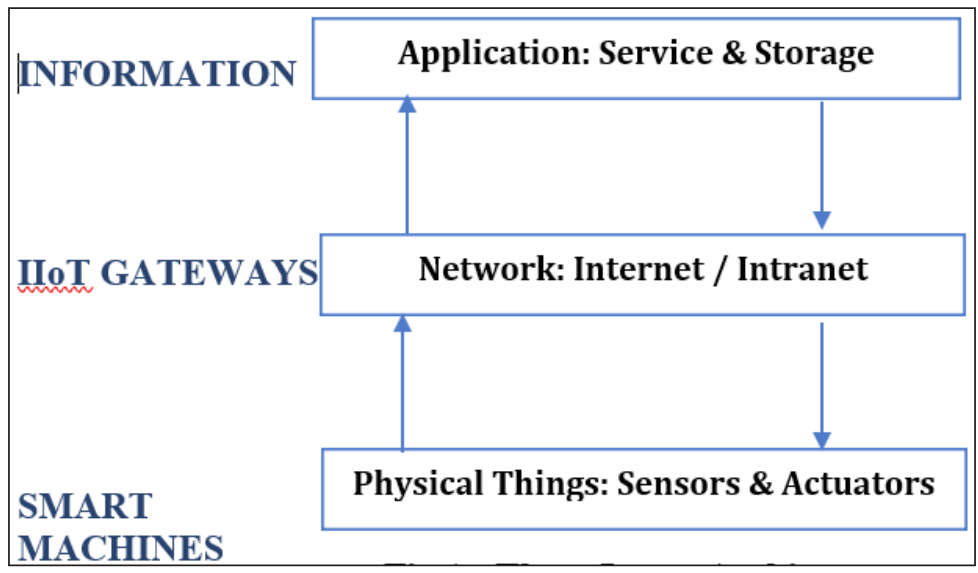

Fig. 1: Three Layer Architecture

The above figure shows the abstract level of IIoT Architecture design with three layers - Physical, Network and Application layer. There are two types of layer models in IIoT solutions (i) Analytical Model and (ii) Data Model. The data models provide a structure to the data while the analytical models are structured to meet industry specific needs The analytical models are trained using a historical data set or using advanced machine learning.

\section{(A) Physical Things : Sensors \& Actuators - Smart Machines}

In the physical layer, some industry-specific devices like Sensors, Interpreters, Translators interfaces with ICS placed in the real-world environment. These devices enable the machines in industry environment as Smart Machines ${ }^{[12]}$. Several heterogenous physical things are deployed in the real-world environment like Sensors for monitoring environment, reading temperature, gauging pressure, proximity, location, smoke, humidity, chemical reaction, gas, and so on. Transient Data Stores, which stores the data temporarily to ensure durability during the system or network failure. Local Processors (data transformation, complex event processing, etc..) are also used to provide data to the application user end from the Physical things.

\section{(B) Network : Internet / Intranet - IIoTGateways}

Network Channels act as a medium to connect and for data transfer between Physical Layer to the application end. The channel can be an Intranet if the smart environment has to be monitored within an industry closed monitoring system, known to be private IIoT channel. The smart environment can be built by connecting and monitoring the smart physical things at different locations of various industries on a production pipeline, there the channel supported through internet, known to be Public IIoT channel. The channels deploy different Network Protocls and API's for connectivity between machine to machine and machine to the application end. These channels interface with heterogeneous sensors and receive unstructured data, a sophisticated gateway is required, called to be IIoT Gateway. This gateway can be part of the middleware architecture - a cloud computing environment. Since the data would be huge, 
unstructured and with high velocity of retrieval, the IIoT can filter the captured data and send the structured data over the Internet to the service end.

\section{(C) Application : Service \& storage-Information}

A collection of large amounts of context information from the Real-world IIoT environment to support different application domains are stored, processed and analysed in cloud computing environments. Some of the popular IoT based cloud environments like Thingworx ${ }^{[13]}$, Xively ${ }^{[14]}$, CISCO IoT cloud, AWS IoT Platform, etc.. supports for IIoT application development. Cantaloupesys ${ }^{[15]}$ supports remote stock tracking service in vending machines, $\mathrm{HiKoB}^{[16]}$ collects real-time measurements for the analysis parameters such as temperature gradients within the road, current outdoor temperatures, moisture, dew and frost points from sensors deployed in roads and provides traffic management, real-time information on traffic conditions, and services for freight and logistics. It is also evident that IoT based cloud platforms are allowed to build their own ecosystems with third party extension support for development and distribution through app store. The most user-end service support is extended through the Mobile Applications which allows users to interact and take or perform actuation tasks from any location at any point of the product development cycle in the industry environment, Mobile-based applications are also support the location-awareness service, i.e., service support based on the geo-graphic location specifically on Smart Enterprises - Transportation and Logistics, Energy and power production, Safety \& Smart Infrastructure support. Wearable devices with gesture-based interactions also plays a significance role in IIoT applications also.

\section{INDUSTRY 4.0 - IIoT INTEGRATED APPLICATIONS}

\section{The integration of IoT technology with industrial automation results in fourth industry revolution - Industry}

4.0 for an objective to attain a high level of productivity with effective operational and maintenance track. Digital optimization of production, Automation with industry environment adaption, Intelligent Data communication for remote action and effective and ease of Human-Machine interaction (HMI) are the major features of industry $4.0^{[21]}$. Industry 4.0 makes a disruptive change in the traditional supply chain process and business models by integrating the IoT enabled services in the industry environment to build a smart industry ecosystem, for achieving the objective of the Industry 4.0.

Fig. 2, shows the integrated IIoT applications for Industry 4.0, builds a smart productive environment, where different types and forms of massive amount of data is generated by the integration of sensor devices and communication technology for smart

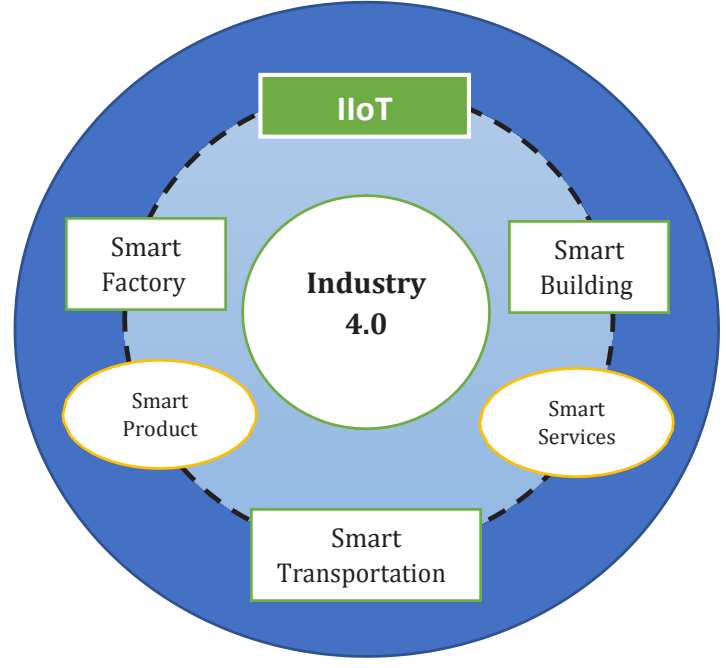

Fig. 2: Industry 4.0 - IIoT integrated Applications 
services and product development in manufacturing sectors. Smart manufacturing are equipping machines with sensors, actuators, microchips and automatic recognition \& detection in support with the computervision based system. Agile software engineering technique is transferred to the manufacturing domain describing an agile factory prototype ${ }^{[18]}$. The developed smart product requires a smart logistics support for transportation by tracking the location and predicting the delivery-time with automated routing applications by sensing the roadway parameters like temperature, mist, humidity etc.. Smart Building ${ }^{[17]}$ combines the Internet, Telecommunication networks, Short distance networks (like Bluetooth, NFC etc..), Broadband networks and Sensor networks to build a smart living environment that ensures the quality of day-to-day life, connected with technology, safety \& security of living place with recognition, detection and prediction systems and effective energy consumption with atmosphere sensed adaptive electric or electronic devices.

\section{CONCLUSION}

This paper presented an overview of the integration of Internet of Things (IoT) and Industrial Internet of Things (IIoT) for the fourth industry revolution - Industry 4.0. This review provides a comparison study on IoT and IIoT and a three-layer IIoT architecture which describes the detailed functional operations and challenges. We have also discussed on the Smart Factory, Smart transportation and Smart Building systems which plays a vital role in the industry 4.0 applications. Further research work needs to be carried out related to IIoT cyber security issues and potential future directions of security threats and attacks that can be addressed in integration with computer-vision and AR/VR based industrial systems.

\section{REFERENCES}

1. A. Zanella, N. Bui, A. Castellani, L. Vangelista and M. Zorzi, 2014. "Internet of Things for Smart Cities", IEEE Internet of Things Journal, 1(1).

2. Li Da XU, Wu He, Shancang L.I., 2014. "Internet of things in industries: a survey", IEEE Transactions on Industrial Informatics, 10(4).

3. J.D. Lin, A.M.K. Cheng and G. Gercek, 2016. "Partitioning Real-Time Tasks with Replications on Multiprocessor Embedded Systems”. IEEE Embedded Systems Letters, 8(4).

4. H. Son, N. Kang, B. Gwak and D. Lee, 2017. "An adaptive IoT trust estimation scheme combining interaction history and stereotypical reputation", $14^{\text {th }}$ IEEE Annual Consumer Communications \& Networking Conference (CCNC), pp. 349-352, Year, 2017.

5. C. Zhu, J.J. Rodrigues, V.C. Leung, L. Shu and L.T. Yang, 2018. "Trust-based communication for the industrial internet of things," IEEE Communications Magazine, 56(2): 16-22.

6. X. Li, D. Li, J. Wan, C. Liu, and M. Imran, “Adaptive transmission optimization in SDN-based industrial internet of things with edge computing," IEEE Internet of Things Journal, 2018.

7. B.M. Lee and H. Yang, "Massive mimo for industrial internet of things in cyber-physical systems," IEEE Transactions on Industrial Informatics, 2017. 
8. L. Lyu, C. Chen, Z. Shanying, and X. Guan, "5g enabled co-design of energy-efficient transmission and estimation for industrial IoT systems," IEEE Transactions on Industrial Informatics, 2018.

9. J. Akerberg, M. Gidlund, and M. Bjorkman, 2011. "Future research challenges in wireless sensor and actuator networks targeting industrial automation," in Proceedings of the $9^{\text {th }}$ IEEE International Conference on Industrial Informatics, 2011, pp. 410-415.

10. C. Gong, 2009. "Human-Machine Interface: Design Principles of Visual Information in Human-Machine Interface Design”, International Conference on Intelligent Human-Machine Systems and Cybernetics Year: 2009, Vol. 2.

11. "Industrial internet reference architecture," http://www.iiconsortium.org/IIRA.htm.

12. J. DeNatale, R. Borwick, P. Stupar, R. Anderson, K. Garrett, W. Morris and J.J. Yao,"MEMS high resolution 4-20 mA current sensors for industrial I/O applications", TRANSDUCERS '03, $12^{\text {th }}$ International Conference on Solid-State Sensors, Actuators and Microsystems. Digest of Technical Papers, Volume: 2, Year 2003.

13. Thing Worx, "Thingworx: Smart systems innovator," Harbor Research, Tech. Rep., 2013, http://www. thingworx.com/ [accessed on: 2013-0822].

14. LogMeIn Inc., "Xively,” 2013, https://xively.com/ [accessed on:201308-22].

15. Cantaloupe Systems, "Seed Platform," 2012, http://www.cantaloupesys. com/ [accessed on:2013-08-22].

16. HiKoB, "Project Grizzly," 2013, http://www.hikob.com/traficexploitation-routiere-its [accessed on: 2013-08-13].

17. V Roblek, M Mesko, A Krapez “A complex view of industry 4.0”, SAGE Open 6(2) (2016).

18. C. Scheuermann, S. Verclas, B. Bruegge, Agile factory - an example of an Industry 4.0 manufacturing process, In: 2015 IEEE $3^{\text {rd }}$ International Conference on Cyber-Physical Systems, Networks and Applications (CPSNA), IEEE, 2015, pp. 43-47. 